\title{
An Analogy between the Properties of "Dark Energy" and Physical Vacuum Consisting of Quantum Harmonic Oscillators Characterized by Zero-Point Energy
}

\author{
Liudmila B. Boldyreva \\ State University of Management, Moscow, Russia \\ Email: boldyrev-m@yandex.ru
}

How to cite this paper: Boldyreva, L.B. (2019) An Analogy between the Properties of "Dark Energy" and Physical Vacuum Consisting of Quantum Harmonic Oscillators Characterized by Zero-Point Energy. Journal of Modern Physics, 10, 20-34. https://doi.org/10.4236/jmp.2019.101003

Received: December 15, 2018

Accepted: January 20, 2019

Published: January 23, 2019

Copyright () 2019 by author(s) and Scientific Research Publishing Inc. This work is licensed under the Creative Commons Attribution International License (CC BY 4.0).

http://creativecommons.org/licenses/by/4.0/ (c) (i) Open Access

\begin{abstract}
In quantum field theory, the physical vacuum, free from magnetic and electric fields (without regard to gravitational energy), is defined not as an empty space but as the ground state of the field consisting of quantum harmonic oscillators (QHOs) characterized by zero-point energy. The aim of this work is to show that such physical vacuum may possess the properties similar to the properties of dark energy: the positive density, the negative pressure, and the possibility of so-called accelerated expansion. In the model discussed, the mass of QHOs determines the positive density of dark energy. The observed electric polarization of physical vacuum in an electric field means the existence of electric dipole moment of QHO, which, in turn, suggests the existence inside the $\mathrm{QHO}$ of a repulsive force between unlike charges compensating the attractive Coulomb force between the charges. The existence of such repulsive force may be treated as the existence of omniradial tensions inside every QHO. In terms of hydrodynamics, it means that the vacuum with this property may be regarded as a medium with negative pressure. The electric dipole-dipole interaction of QHOs under some condition may result in the expansion of physical vacuum consisting of QHOs. It is shown also that the physical vacuum consisting of QHOs is a luminiferous medium, and based on this concept the conditions are discussed for the emergence of invisiblity of any objects (in particular, dark matter). The existence of luminiferous medium does not contradict the second postulate of special relativity (the principle of constancy of the velocity of light in inertial systems), if to take into account the interaction of photons with QHOs and with virtual photons (the virtual particles pairs) created by quantum entities that constitute the inertial systems.
\end{abstract}




\section{Keywords}

Dark Energy, Dark Matter, Zero-Point Energy, Quantum Harmonic Oscillator, Cosmic Microwave Background

\section{Introduction}

According to contemporary cosmological models, near 70 percent of the total mass-energy of the universe is in the form of so-called dark energy or "quintessence" which is characterized by the homogeneous distribution of positive density, by negative pressure and by the possibility of accelerated expansion [1] [2] [3]. At present, there is no generally accepted physical model of dark energy. In this work, it is shown that the physical vacuum consisting of quantum harmonic oscillators (hereafter called QHOs) characterized by zero-point energy may have all the properties of dark energy.

In quantum field theory, the physical vacuum, free from magnetic and electric fields (without regard to gravitational energy), is defined not as an empty space but as the ground state of the field consisting of QHOs characterized by non-zero energy equal to $h v / 2$. The concept of zero-point energy was developed in Germany in 1913 by a group of physicists, including M. Planck, A. Einstein, and O. Stern [4], using the formula derived by Planck [5] for energy $\varepsilon$ of atomic oscillator vibrating with frequency $v: \varepsilon=h v / 2+h v /(\exp (h v /(k T))-1)$, where $h$ is the Planck constant, $k$ is the Boltzmann constant, $T$ is temperature. The properties of the physical vacuum consisting of QHOs as a continuum are determined by the properties of QHOs [6]. The mass of QHOs associated with their energy determines the positive density of the physical vacuum. The observed electric polarization of physical vacuum in an electric field means the existence of electric dipole moment of $\mathrm{QHO}$, which, in turn, suggests the existence of a repulsive force between unlike charges inside the QHO, which may be treated as the existence of omniradial tensions inside the QHO. In terms of hydrodynamics, it means that the vacuum with this property may be regarded as a medium with negative pressure [7]. The electric dipole-dipole interaction of QHOs may result in the so-called accelerated expansion of the physical vacuum consisting of QHOs. It is shown as well that the physical vacuum consisting of QHOs is a luminiferous medium, and based on this concept the conditions are discussed for the emergence of invisiblity of any objects (in particular, dark matter). Due to possibility of emergence of spin supercurrent between QHOs the background electro-magnetic emission may exist in the physical vacuum consisting of QHOs. The cosmic microwave background (CMB) may be such an emission.

The work below consists of three sections. In Section 2, the properties of physical vacuum consisting of QHOs are considered, in particular: the connection of speed of motion of such vacuum and magnetic phenomena. In Section 3, the following is discussed: the emergence of the wave-vortex-spin (electromagnetic) 
process in the physical vacuum, equalizing the speed of light in inertial systems, the condition of disappearance of wave-vortex-spin process (the condition of invisibility of objects). In Section 4, the electric dipole-dipole interaction of QHOs, which results in a change in the distance between these QHOs, is discussed.

\section{The Properties of Physical Vacuum Consisting of QHOs}

\subsection{The Equation Describing the Physical Vacuum, Consisting of QHOs, in a Stationary State}

Based on the characteristics of QHOs mentioned in Introduction, the following conclusions can be made about the properties of the physical vacuum consisting of QHOs (the vacuum being in a stationary state): the positive density created by the mass of QHOs and negative pressure. As it will be shown in Section 4, the density of the physical vacuum is slightly dependent on speed $u$ at small $u / c$ ( $c$ is the speed of light). The negative pressure is due to the existence of electric dipole moment of $\mathrm{QHO}$, that is, the existence of a repulsive force between unlike charges inside the $\mathrm{QHO}$, compensating the attractive Coulomb force between these charges. The existence of such repulsive force may be treated as the existence of omniradial tensions inside the QHO [7]. Taking into account the dissipation-free motion of celestial bodies, such as the planets of the solar system, that is the absence of shear viscosity, it may be assumed that the physical vacuum consisting of QHOs in a stationary state is analogous to ideal incompressible liquid, and can be described by the following Equation [7] [8]:

$$
\rho u^{2} / 2-p=\text { const },
$$

where $\rho$ and $p$ are respectively the density and pressure of the physical vacuum consisting of QHOs.

\subsection{The Connection of the Speed of Motion of Physical Vacuum Consisting of QHOs with Magnetic Phenomena}

It is shown in [7], that there is a complete analogy between the structures of formulas describing the magnetic interactions of current-carrying wires and the structures of formulas describing the interactions of vortices in an ideal incompressible liquid with positive density and negative pressure, that is, in the liquid described by Equation (1) in a stationary case.

Let us deduce the relationship between the speed of QHOs and magnetic induction by comparing the characteristics of the magnetic field and both force and kinematic characteristics of physical vacuum consisting of QHOs [7].

The magnetic induction $\boldsymbol{B}$ generated by a loop with current $I$ [9] is determined by the Biot-Savart law and in the CGSE system of units it is determined as: $\boldsymbol{B}=\frac{I}{c} \int_{L^{\prime}} \frac{\mathrm{d} \boldsymbol{l} \times \boldsymbol{r}}{r^{3}}$, where $L^{\prime}$ is the length of the loop, $\mathrm{d} \boldsymbol{l}$ is the wire element, $\boldsymbol{r}$ is a radius vector from $\mathrm{d} \boldsymbol{l}$ to the point of observation. The field of velocities $\boldsymbol{u}$ generated by a closed vortex line having circulation $\Gamma$ along an arbitrary loop enclosing the vortex line is defined [7] as: $\boldsymbol{u}=\frac{\Gamma}{4 \pi} \int_{L^{\prime}} \frac{\mathrm{d} \boldsymbol{l} \times \boldsymbol{r}}{r^{3}}$, where $\mathrm{d} \boldsymbol{l}$ is an in- 
finitesimal vector element of the vortex line, $L^{\prime}$ is the length of the line. Equating the expressions for $\boldsymbol{B}$ and $\boldsymbol{u}$ we obtain the relationship between $\Gamma$ and I:

$$
\Gamma=\boldsymbol{I} \sqrt{4 \pi} /(c \sqrt{\rho}) .
$$

Thus, Equation (2) establishes a relationship between the current and circulation of vortex line produced by moving electric charges that form the current (in detail see Section 2.3).

The force $F_{\Gamma}$ acting on a unit length of either of the two infinite mutually parallel vortex lines having the same values of circulation $\Gamma$ equals [7]: $F_{\Gamma}=\rho \Gamma^{2} /\left(2 \pi r_{w}\right)$, where $r_{w}$ is the distance between the vortex lines with circulation $\Gamma$. The force $F_{I}$ acting on a unit length of either of the two infinite mutually parallel current-carrying wires having the same values of current $I$ (in the CGSE system of units) equals [9]: $F_{I}=2 I^{2} /\left(r_{w} c^{2}\right)$, where $r_{w}$ is here the distance between the current-carrying wires. Equating the expressions for $F_{\Gamma}$ and $F_{I}$ and taking into account Equation (2), we obtain.

$$
\boldsymbol{B}=\boldsymbol{u} \sqrt{4 \pi \rho} .
$$

There is indirect experimental evidence of validity of Equation (3). The term "indirect" is used because in the experiments in question the evidence refers to the neutrino whose properties are mysterious in some respects. At present, the concept of massive neutrino with its magnetic moment aligned with its spin is considered to be most acceptable to physicists. From observations it follows that the spin of a left-handed neutrino moving relative to the "cosmic" vacuum is oriented opposite to its velocity $\boldsymbol{v}$, according to Equation (3), this motion is equivalent to placing the neutrino in the magnetic field with magnetic induction $\boldsymbol{B}=-\boldsymbol{v} \sqrt{4 \pi \rho}$. It is in accordance with that in an external magnetic field (whose magnetic induction in the experiments was much greater than that of the Earth) the neutrino spin got oriented in the magnetic induction direction [10] [11].

\subsection{Electric Current as a Vortex Line in the Physical Vacuum Consisting of QHOs}

According to postulates of quantum mechanics, a quantum entity (its characteristics are determined by the wave function) that is a singularity in electric or magnetic fields (electric charge or/and magnetic dipole) creates a virtual photon (pair of virtual particles) [12] [13]. The virtual photon is characterized by spin $\boldsymbol{S}_{v}$ precessing with frequency $\boldsymbol{\omega}_{v}$ (that is, the virtual photon is a spin vortex), mass $m_{v}$, electric dipole moment $\boldsymbol{d}_{v}$ and circulation $\Gamma_{v}$ (Figure 1). Let us consider the properties of virtual photons in detail.

Spin. As the virtual photon is a spin vortex, then by the analogy with (real) photon the following holds [8] [14] with respect to spin $\boldsymbol{S}_{v}$ and electric dipole moment $\boldsymbol{d}_{v}$ :

$$
\boldsymbol{d}_{v} \uparrow \downarrow \boldsymbol{S}_{v} .
$$

Taking into account that electric field $\boldsymbol{E}_{v}$ inside the electric dipole is antiparallel 


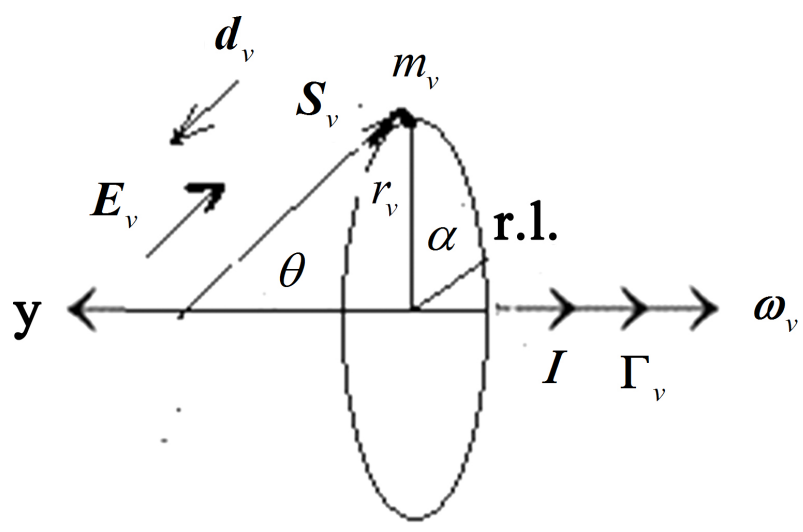

Figure 1. The characteristics of a virtual photon created by electron: $\boldsymbol{S}_{v}$ is spin, $\boldsymbol{d}_{v}$ is the electric dipole moment, $\boldsymbol{E}_{v}$ is the electric field inside the electric dipole, $\Gamma_{v}$ is circulation, $\omega_{v}$ is the precession frequency, $I$ is electric current, $y$ is the velocity of electron, $\theta$ is the deflection angle between $\boldsymbol{\omega}_{v}$ and $\boldsymbol{S}_{v}, \alpha$ is the precession angle, $r_{v}$ is the radius of circle by which mass $m_{v}$ performs circulation motion, on the premise that the mass is point-like, r.l. is a reference line.

to its electric dipole moment, $\boldsymbol{E}_{v} \uparrow \downarrow \boldsymbol{d}_{v} \quad[9]$, we have:

$$
\boldsymbol{E}_{v} \uparrow \uparrow \boldsymbol{S}_{v} .
$$

Precession frequency. As follows from the study by L. Boldyreva [15], the precession frequency $\omega_{v}$ of spin of the virtual photon (pair of virtual particles) created by a quantum entity equals the frequency $\omega_{q}$ of the wave function of the entity: $\omega_{q}=\omega_{v}$. In Schrodinger's wave function the value of $\omega_{q}$ is determined by energy $U_{q}$ of quantum entity: $\omega_{q}=U_{q} / \hbar$, consequently:

$$
\omega_{v}=U_{q} / \hbar \text {. }
$$

Let us consider the case where energy $U_{q}$ of quantum entity equals its kinetic energy:

$$
U_{q}=m_{q} y^{2} / 2
$$

where $m_{q}$ and $y$ are respectively mass and speed of the quantum entity. Then from Equations (6) and (7) it follows:

$$
\omega_{v}=m_{q} y^{2} /(2 \hbar) \text {. }
$$

If a virtual photon is created by electrically charged quantum entity, then electric field $\boldsymbol{E}_{q}$ of this entity acts on the virtual photon as on an electric dipole; the emerging moment $\boldsymbol{M}_{q}$ is determined [9] as $\boldsymbol{M}_{q}=\boldsymbol{d}_{v} \times \boldsymbol{E}_{q}$. Because of the action of moment $\boldsymbol{M}_{q}$, the orientation of $\boldsymbol{\omega}_{v}$ shall be determined by the sign of the quantum entity. Taking into account that direction of velocity $y$ is the single preferential direction for virtual photon, we may assume:

$$
\omega_{v} \uparrow \uparrow \eta \boldsymbol{y}
$$

where $\eta=1$ for positively charged quantum entity and $\eta=-1$ for negatively charged quantum entity, that is:

$$
\omega_{v} \uparrow \uparrow \boldsymbol{I}
$$


where $I$ is the current created by the moving charged quantum entity.

Electric dipole moment. The electric dipole moment of virtual photon $d_{v}$ may be determined [14] as:

$$
d_{v}=q_{v} \lambda_{q}
$$

where $q_{v}$ is the charge of every virtual particle in the virtual photon; $\lambda_{q}$ is the wave function wavelength of quantum entity creating the virtual photon and it is determined [16] as:

$$
\hbar_{q}=\hbar /\left(m_{q} y\right)
$$

Let us deduce the expression for $d_{v}$ for the virtual photon created by an electron, assuming that the specific charge of the virtual particle in the virtual photon is proportional to the specific electron charge $e / m_{e}$ ( $e$ and $m_{e}$ are respectively the electric charge and mass of electron). Note that the experiments conducted by W. Kaufmann on deflection of beta-rays emitted by radium make one believe that the mass of electron is purely of electromagnetic nature [17]. Thus $q_{v}=m_{v} e /\left(2 m_{e}\right)$; the virtual photon mass $m_{v}$, taking into account Equation (7) and classical relationship between mass and energy, is determined as:

$$
m_{v}=m_{q} y^{2} /\left(2 \cdot c^{2}\right) \text {. }
$$

Using the above considered expression for $q_{v}$, Equation (13) and the expression for Bohr's magneton $\left(\mu_{B}=e \cdot \hbar\left(2 \cdot m_{e} \cdot c\right)\right)$ in Equation (11) we obtain:

$$
d_{v}=\frac{\mu_{B} \cdot y}{2 \cdot c} \text {. }
$$

If for the virtual particles pair created by electron moving at velocity $y(y \ll c)$ it holds that $\boldsymbol{d}_{v} \uparrow \uparrow \boldsymbol{y}$, then from Equation (14) it follows that in the electric field $\boldsymbol{E}$ the moment $\boldsymbol{M}$ acting on the electric dipole, $\boldsymbol{M}=\boldsymbol{d}_{v} \times \boldsymbol{E}$, is determined as: $\boldsymbol{M}=\frac{\mu_{B}}{2 \cdot c}(\boldsymbol{y} \times \boldsymbol{E})$; the right side of expression for $\boldsymbol{M}$ is the same as that for maximum value of the spin-orbit interaction energy of the electron in a hydrogen atom: $\left(U_{s-o}\right)_{\max }=\left|\frac{\mu_{B}}{2 \cdot c}(\boldsymbol{y} \times \boldsymbol{E})\right|$. Thus at $y \ll c$ the condition $\boldsymbol{d}_{v} \uparrow \uparrow \boldsymbol{y}$ holds true. Taking into account conditions (4) and (9), and that for photon spin $\boldsymbol{S}_{p h}$ the following is valid: $\boldsymbol{S}_{p h} \perp \boldsymbol{c}$ ( $c$ is the velocity of light) [14] [18], we may introduce the following equation for deflection angle $\theta$ between the precession frequency of virtual photon and its spin:

$$
\sin \theta=y / c \text {. }
$$

The circulation. Using the above-considered expressions for characteristics of virtual photon let us analyze the circulation motion of mass $m_{v}$ that is performed as a result of precession motion of spin $\boldsymbol{S}_{v}$. The circulation $\Gamma_{v}$ characterized by this circulation motion is defined [7] as:

$$
\Gamma_{v}=2 \pi \omega_{v} r_{v}^{2}
$$

where $r_{v}$ is the radius of circle by which mass $m_{v}$ performs circulation motion, on the premise that the mass is point-like. From Equations (8), (10) and (16) it 
follows: $\Gamma_{v}=\boldsymbol{I} \pi m_{q} y^{2} r_{v}^{2} /(I \hbar)$. Thus the moving charged quantum entity creates a vortex line in the physical vacuum consisting of QHOs with circulation directed along the current created by motion of this quantum entity. The circulation $\Gamma_{I}$ created by electric current $I$ is determined as sum of $\Gamma_{v}$ for all charged quantum entities that constitute the electric current. It should be noted that virtual photons created by charged quantum entities, the speed $y$ of which is directed along the same axis, have equal precession angles $\alpha$. This equalization of precession angles $\alpha$ along the vortex line created by electric current is performed by spin supercurrent. Let us consider it in detail.

Spin supercurrent. Spin supercurrent was discovered while investigating the characteristics of superfluid ${ }^{3} \mathrm{He}-\mathrm{B}$ [19] [20] [21]. For example, the value of spin supercurrent $j_{z}$ in the direction of orientation (axis $z$ ) of precession frequencies of spins of ${ }^{3} \mathrm{He}$ atoms is determined as follows:

$$
j_{z}=-g_{1} \partial \alpha / \partial z-g_{2} \partial \theta / \partial z
$$

where $\alpha$ is the precession angle (phase), $\theta$ is the deflection angle. The spin supercurrent tends to equalize the respective characteristics of spins of interacting spin structures: angles (phases) of precession and angles of deflection. For example, after action of spin supercurrent $J_{z}$ between spin structures with precession angles $\alpha_{1}$ and $\alpha_{2}$, and deflection angles $\theta_{1}$ and $\theta_{2}$ the following takes place:

$$
\begin{gathered}
\left|\alpha_{1}-\alpha_{2}\right|>\left|\alpha_{1}^{\prime}-\alpha_{2}^{\prime}\right|, \\
\left|\theta_{1}-\theta_{2}\right|>\left|\theta_{1}^{\prime}-\theta_{2}^{\prime}\right|,
\end{gathered}
$$

where $\alpha_{1}^{\prime}$ and $\alpha_{2}^{\prime}$ are respectively the values of precession angles $\alpha_{1}$ and $\alpha_{2}$ after the action of spin supercurrent; $\theta_{1}^{\prime}$ and $\theta_{2}^{\prime}$ are respectively the values of deflection angles $\theta_{1}$ and $\theta_{2}$ after the action of spin supercurrent.

Virtual photon and QHOs. The photon may decay into a pair of oppositely charged particles in the electric field of heavy nuclei [16]. In this case, the total spin of emerging particles equals the photon spin, which suggests that the principle of conservation of angular momentum holds true in the physical vacuum where the photon emerges. Consequently, the creation of virtual photons having precessing spin by a quantum entity while saving the value of its own spin testifies that the spin of virtual photon is formed by spins of particles that constitute the physical vacuum: in particular, by spins of QHOs. (The analogous conclusion may be made for "real" photons as well while analyzing the Cherenkov effect [22]: the production of photons having spin by an electron moving at a superluminal speed while saving the value of its own spin. It should be noted that spin of "real" photon in pure state performs precession motion with frequency of photon.) Thus QHO as a harmonic oscillator having precessing spin might be classified as a spin vortex, and the frequency of oscillations $\Omega_{\mathrm{Pно}}$ may be the precession frequency of spin of QHO. Consequently, QHO may possess the properties of such spin vortex as the virtual photon and the expressions similar to (4)-(5), (9) and (14)-(15) hold true as well for QHO, that is: 


$$
\begin{gathered}
\boldsymbol{d}_{\mathrm{QHO}} \uparrow \downarrow \boldsymbol{S}_{\mathrm{QHO}}, \\
\boldsymbol{E}_{\mathrm{QHO}} \uparrow \uparrow \boldsymbol{S}_{\mathrm{\textrm {HO }}}, \\
\boldsymbol{\Omega}_{\mathrm{PHO}} \| \boldsymbol{u}, \\
d_{\mathrm{QHO}} \approx \gamma \cdot u, \\
\sin \theta=u / c,
\end{gathered}
$$

where $\boldsymbol{d}_{\mathrm{QHо}}$ is the electric dipole moment of QHO, $\boldsymbol{S}_{\mathrm{QHO}}$ is spin of QHO, $u$ is the speed of QHO, $\gamma$ is a proportionality factor, $\theta$ is the deflection angle between $S_{\mathrm{QHO}}$ and frequency $\Omega_{\mathrm{QHO}}$ of its precession.

\section{The Wave-Vortex-Spin Process in the Physical Vacuum Consisting of QHOs}

\subsection{The Equation Describing the Wave-Vortex-Spin Process in the Physical Vacuum Consisting of QHOs}

Due to the existence of interactions of QHOs (the electric dipole-dipole interaction and that owing to spin supercurrents), the physical vacuum consisting of QHOs should feature the rotational viscosity, which manifests itself in a nonstationary case, in particular, the transformation of macrorotation in microrotation and vice versa. It is shown that the wave-vortex-spin process may arise in the physical vacuum (see also [23]). It should be noted that the vortices in the physical vacuum may terminate in the bulk of the physical vacuum due to complete transfer of the angular momentum of vortex to intrinsic motions (to intrinsic degrees of freedom) of the physical vacuum.

\section{The first Equation describing the wave-vortex-spin process}

Due to conservation of angular momentum in the physical vacuum, the Einstein-de Haas effect takes place in this vacuum [24]: a change of spin $S$ $(\partial \boldsymbol{S} / \partial t \neq 0)$ of a unit volume of physical vacuum consisting of QHOs results in the rotation of the vacuum ( $\operatorname{curl} \boldsymbol{u} \neq 0)$. That is the following holds true:

$$
\partial \boldsymbol{S} / \partial t=-\left(1 / k_{1}\right) \cdot \text { curlu },
$$

where $t$ is time, $k_{1}>0$ is a proportionality factor.

The second Equation describing the wave-vortex-spin process

According to Equations (22) and (24), at the emergence of $\partial \boldsymbol{u} / \partial t$ in the physical vacuum consisting of QHOs the following cases may take place:

1 ) at a change in the direction of velocity $\boldsymbol{u}$, the precession motion of $S$ relative to a new direction of $\boldsymbol{u}$ arises;

2 ) at a change of only the value of $u$, the angle $\theta$ changes.

In both cases, a change in deflection angle $\theta$ takes place, which, in turn, results in emergence of curlS. That is, the following equation should be taken to be true:

$$
\partial \boldsymbol{u} / \partial t=k_{2} \operatorname{curl} \boldsymbol{S}
$$

where $k_{2}>0$ is a proportionality factor. To make it clear that Equations (25) and (26) describe the wave-vortex-spin process in the physical vacuum consist- 
ing of QHOs let us introduce the following factor $\chi$ :

$$
\chi=\sqrt{k_{2} / k_{1}}
$$

Using Equation (27) in Equations (25) and (26) we obtain:

$$
\begin{aligned}
& \frac{\partial\left(\chi k_{1} \boldsymbol{S}\right)}{\partial t}=-\chi \text { curlu, } \\
& \frac{\partial \boldsymbol{u}}{\partial t}=\chi \operatorname{curl}\left(\chi k_{1} \boldsymbol{S}\right) .
\end{aligned}
$$

The dimension of factor $\chi$ is the same as that of speed. The Equations (28) and (29) describe the wave-vortex-spin process in which transformation of energy is performed as follows: the specific kinetic energy of motion of physical vacuum $\rho u^{2} / 2$ transforms into energy $W_{S}$ of spin system of the vacuum that creates the spin vortex; in turn, the energy $W_{S}$ transforms into the kinetic energy of motion of the physical vacuum. The energy $W_{S}$ may be detailed if to introduce the following notation in Equations ((28), (29)):

$$
\boldsymbol{Y}=-k_{1} \chi \boldsymbol{S} \text {. }
$$

Then

$$
\begin{aligned}
& \frac{\partial \boldsymbol{Y}}{\partial t}=\chi \cdot \operatorname{curl} \boldsymbol{u}, \\
& \frac{\partial \boldsymbol{u}}{\partial t}=-\chi \cdot \operatorname{curl} \boldsymbol{Y} .
\end{aligned}
$$

Let us consider the physical meaning of variable $Y$, which, according to (30), is directed oppositely to $S$, is proportional to the magnitude of $S$ and has the dimension of velocity. It may be supposed that $Y$ is a velocity of motion of positive charges of QHO (simultaneously, negative charges of $\mathrm{QHO}$ move at velocity- $Y$ ), see Figure 2. Such motions result in creation of electric field $E$ inside QHO. Then $W_{S}$ is the kinetic energy of motion of charges inside QHO at speed $Y$, that is in the wave-vortex-spin process the transformation of energy is performed as follows: the specific kinetic energy of motion of physical vacuum $\rho u^{2} / 2$ around the vortex transforms into specific kinetic energy of motion of physical vacuum $\rho Y^{2} / 2$ inside the vortex.

As electric field $E$ emerges inside QHO due to motion of charges inside QHO at speed $Y$, the specific kinetic energy of this motion $\left(\rho Y^{2} / 2\right)$ transforms in the specific energy of emerging electric field $E\left(E^{2} /(8 \pi)\right)$. It follows from equality of those energies:

$$
\boldsymbol{E}=-\boldsymbol{Y} \sqrt{4 \pi \rho}
$$

Using Equations (3) and (33) in Equations (31) and (32), we obtain the equations describing the electromagnetic process:

$$
\begin{aligned}
& \frac{\partial \boldsymbol{E}}{\partial t}=\chi \cdot \operatorname{curl} \boldsymbol{B}, \\
& \frac{\partial \boldsymbol{B}}{\partial t}=-\chi \cdot \operatorname{curl} \boldsymbol{E} .
\end{aligned}
$$


Consequently, the physical vacuum consisting of QHOs may be considered as a luminiferous medium.

Note. In emerging electric field $\boldsymbol{E}$ the moment $\boldsymbol{M}_{\boldsymbol{E}}$ acts on QHO as on electric dipole: $\boldsymbol{M}_{\boldsymbol{E}}=\boldsymbol{d}_{\mathrm{\textrm {HO }}} \times \boldsymbol{E}$. Simultaneously, according to condition (20), $\boldsymbol{M}_{\boldsymbol{E}}$ acts as well on spin $\boldsymbol{S}_{\text {Qно }}$ of QHO. Thus in an electric field the electric and spin polarizations of physical vacuum consisting of QHOs characterized by zero-point energy emerge.

\subsection{The Equalization of Speed of Wave-Vortex-Spin Process in Inertial Systems}

The existence of a luminiferous medium does not contradict the second postulate of special relativity; this principle of the constancy of the velocity of light states: "in all inertial systems the velocity of light has the same value when measured with length-measures and clocks of the same kind" [25].

In this Section (see also ref. [14]), it will be shown that this postulate may be due to the interaction of the photons with QHOs constituting the physical vacuum and with the virtual photons (virtual particles pairs) created by quantum entities that constitute the inertial system (and determine, in fact, its inertial properties). One of the first works containing the physical interpretation of the equalization of the speed of light in inertial systems to a definite value is the work by Fox [26]. The studies by Fox were directed at supporting the Ritz emission theory, according to which the fundamental constant $c$ is the speed of light with respect to the source in the vacuum and the Galilean addition of velocities holds [27]. Fox used the extinction theorem of Ewald and Oseen [28]. The theorem states that if an incident electromagnetic wave traveling at a speed $c$ appropriate to vacuum enters a dispersive medium, its fields are cancelled by part of the fields of the induced dipoles (macroscopically, by the polarization) and replaced by another wave propagating with a phase velocity characteristic of the medium. The incident wave is extinguished by interference and replaced by another wave. The motion of the source and the speed of light relative to it are irrelevant in this theorem. There are, however, some experiments that are not explained by the extinction theorem, for example the experiment performed at CERN, Geneva, in 1964 [29]. In this experiment, photons were produced by the source moving at speed of $0.99975 c$ relative to the measurement devices. Photons' speed was measured by time of flight over paths up to 80 meters; within the experimental error it was found that the speed of the photons was equal to $c$ relative to the same measurement devices. The extinction theorem, in which the interaction of a photon and a medium takes place due to the magnetic and electric components of photon, does not explain the results of the experiment. The equalization of the speed of light found in experiments indicates the existence of some other interactions. In particular, it is necessary to take into account the interaction of photons with QHOs that constitute the physical vacuum and with virtual photons (the virtual particles pairs) created by quantum entities that constitute the inertial systems. 


\subsection{The Condition of Disappearance of Wave-Vortex-Spin Process}

Equation (28) describing the wave-vortex-spin process contains $\partial \boldsymbol{S} / \partial t$. Consequently, this process could not spread in the region where the orientation of spins of QHOs that constitute the physical vacuum cannot change, i.e. spins can be considered to be "frozen":

$$
\partial S / \partial t \equiv 0
$$

This may take place, for example, in the following cases: 1) at the emergence of spin supercurrents causing a definite orientation of spins and suppressing any disturbances producing a change in the orientation of spins; 2) at rotation of physical vacuum consisting of QHOs, which due to the Barnett effect [30] creates a definite orientation of spins of $\mathrm{QHOs}$ that constitute this vacuum.

One of the most striking examples demonstrating the effect of visibility loss is a series of experiments conducted by J. Searl in 1940-1950 [31]. In the experimental setup a rotating nonlinear magnetic field could be created. At the critical value of speed of rotation the invisibility of the setup is observed. This may be interpreted as follows: according to Equation (3), the rotation of magnetic field means the rotation of physical vacuum consisting of QHOs and consequently, due to the effect of Barnett, gives rise to a definite orientation of spins of QHOs that constitute this vacuum.

In the standard Lambda-CDM model of cosmology, near $27 \%$ of the total mass-energy of the universe consist of dark matter [32]. At present, the invisibility is explained in particular by that the strong gravitation field of the matter does not allow photons to leave the location of the matter. The model of physical vacuum consisting of QHOs considered in this work accounts for the invisibility by emergence of "freezing" of spins (Equation (36)) of those QHOs at the location of this matter. This "freezing" may take place as a result of rotation of dark matter and due to low temperature characterizing dark matter. Let us consider the influence of temperature in detail.

The temperature $T$ determines the velocity $y(y=\sqrt{2 K T / m})$ of thermal chaotic motion of quantum entities (with mass $m$ ) that constitute "dark" matter and consequently the velocity of motion of virtual photons (virtual particles pairs) created by those entities. The speed of quantum entities influences the following characteristics of virtual photons: the value of the deflection angle, see Equation (15); the direction and the value of precession frequency $\omega_{v}$, see Equations ((8), (9)). As the virtual photon is a vortex in the physical vacuum consisting of QHOs, the changes in characteristics of virtual photons mean the changes in the characteristics of QHOs as well. Thus, the thermal chaotic motion may prevent the "freezing" of spins of QHOs that constitute the physical vacuum.

\section{The Electric Dipole-Dipole Interaction of QHOs That Constitute the Physical Vacuum}

Let us consider the projections of electric dipole moment of $\mathrm{QHO}, \boldsymbol{d}_{\mathrm{QHO}}$, on the 
direction of velocity $\boldsymbol{u}$ of $\mathrm{QHO},\left(d_{\mathrm{QHO}}\right)_{u}$, and on the direction perpendicular to $\boldsymbol{u},\left(d_{\mathrm{QHO}}\right)_{\perp u}$, see Figure 2. According to conditions (20) and (22), the projections $\left(d_{\mathrm{QHO}}\right)_{u}$ and $\left(d_{\mathrm{QHO}}\right)_{\perp u}$ are determined as:

$$
\begin{gathered}
\left(d_{\mathrm{QHO}}\right)_{u}=d_{\mathrm{QHO}} \sqrt{1-\sin ^{2} \theta}, \\
\left(d_{\mathrm{QHO}}\right)_{\perp u}=d_{\mathrm{QHO}} \sin \theta .
\end{gathered}
$$

According to Equations (17)-(19), spin supercurrent equalizes both the precession angles $\alpha$ and the deflection angles $\theta$ of QHOs, whose precession frequencies $\Omega_{\text {рно }}$ and velocities $\boldsymbol{u}$ are directed along the same axis $\boldsymbol{z}$ (see Figure 2). Consequently: first, the components $\left(d_{\mathrm{QHO}}\right)_{\perp u}$ of QHOs moving along the same axis $\boldsymbol{z}$ are parallel to each other, and, secondly, these QHOs have equal components $\left(d_{\mathrm{QHO}}\right)_{\perp u}$ and equal components $\left(d_{\mathrm{QHO}}\right)_{u}$ respectively. As a result, the attractive force $F_{\mathrm{QHO}_{\|}}$and repulsive force $F_{\mathrm{QHO}_{\perp}}$ act between those QHOs [9]. According to Equations (24) and (37)-(38), we have:

$$
\begin{gathered}
F_{\mathrm{QHO} \|}=\frac{6 d_{\mathrm{QHO}}^{2}\left(1-u^{2} / c^{2}\right)}{r^{4}}, \\
F_{\mathrm{QHO}_{\perp}}=\frac{3 d_{\mathrm{QHO}}^{2} u^{2}}{c^{2} r^{4}},
\end{gathered}
$$

where $r$ is the distance between the QHOs. The expression for resulting force $F_{\mathrm{QHO}}=F_{\mathrm{QHO}_{\|}}-F_{\mathrm{QHO}_{\perp}}$ with taking into account Equations (39) and (40) may be written in the form: $F_{\mathrm{QHO}}=3 d_{\mathrm{QHO}}^{2}\left(2-3 u^{2} / c^{2}\right) / r^{4}$. Using Equation (23), $F_{\mathrm{QHO}}$ may be expressed in the form:

$$
F_{\mathrm{QHO}}=3 \gamma^{2}\left(2 u^{2} / c^{2}-3 u^{4} / c^{4}\right) / r^{4} .
$$

Consequently, that force $F_{\mathrm{QHO}}$ is repulsive under condition $u>c \sqrt{2 / 3}$.

Let us estimate the influence of components of force $F_{\mathrm{QHO}}$ on the density of the physical vacuum consisting of QHOs characterized by zero-point energy. The force component $F_{\mathrm{QHO}_{\|}}$, being an attractive force, tends to decrease the distance between QHOs moving along the same axis $\boldsymbol{Z}$ and consequently to increase the concentration of QHOs in the physical vacuum. The force component
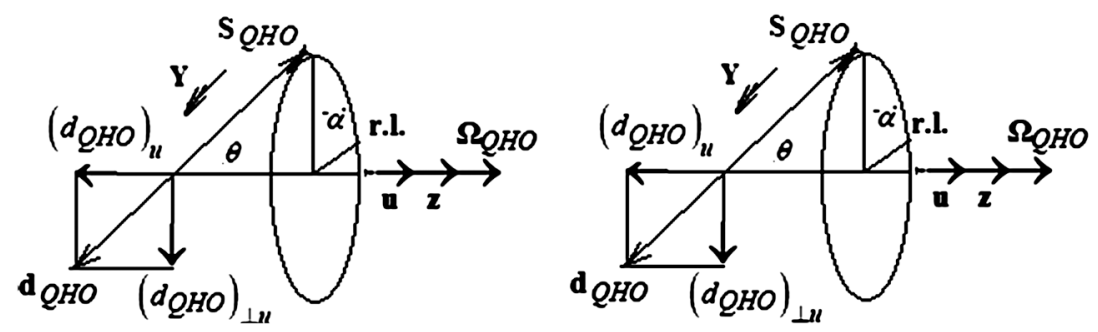

Figure 2. The characteristics of quantum harmonic oscillators (QHOs): $\boldsymbol{S}_{\text {оно }}$ is spin, $\boldsymbol{d}_{\text {Оно }}$ is the electric dipole moment, $\Omega_{\mathrm{Pно}}$ is the frequency of spin, $\left(d_{\text {Рно }}\right)_{\perp u}$ is the projection of $\boldsymbol{d}_{\text {Оно }}$ on the direction perpendicular to velocity $\boldsymbol{u},\left(d_{\text {Оно }}\right)_{u}$ is the projection of $\boldsymbol{d}_{\text {Оно }}$ on the direction $\boldsymbol{u}, \theta$ is the deflection angle, $\alpha$ is the precession angle, r.l. is a reference line, $\boldsymbol{Y}$ is the velocity of positive charges that constitute QHO. 
$F_{\mathrm{QHO}_{\perp}}$, being a repulsive force, tends to increase the distance between QHOs moving along the same axis $z$ and consequently to decrease the concentration of QHOs in the physical vacuum. It follows from Equation (41) that in the first order of $\beta=u / c$ the resulting force $F_{\mathrm{QHO}}$ and consequently the concentration of $\mathrm{QHOs}$ does not depend on the speed of physical vacuum. Since there is no information available on the connection of mass of $\mathrm{QHO}$ with the speed of the latter, it may be assumed that only the concentration of QHOs determines the dependence of density of physical vacuum on the speed. And consequently it may be assumed that the density does not depend on the speed of physical vacuum at small $\beta$.

\section{Discussion. Cosmic Microwave Background (CMB)}

The QHOs that constitute the physical vacuum have precessing spin. Spin supercurrent emerging between QHOs influences the characteristics of the precession (the angles of precession and deflection) changing thus the orientation of spin in space. According to Equations ((28), (29)), this means the possibility of emergence in the physical vacuum of wave-vortex-spin process that, according to Equations ((34), (35)), is also an electromagnetic process. Thus, the background electro-magnetic emission may exist in the physical vacuum consisting of QHOs. The cosmic microwave background (CMB) may be such an emission.

\section{Conclusions}

The properties of physical vacuum consisting of quantum harmonic oscillators (QHOs) characterized by zero-point energy are identical to the properties of dark energy, i.e.:

- the positive density associated with mass of QHOs;

- the negative pressure caused by that in a QHO a separation of the substance of vacuum into positive and negative charges takes place;

- the possibility of increase in distance between QHOs, i.e. of the "expansion" of physical vacuum; it is due to electric dipole-dipole interaction of QHOs;

- the physical vacuum consisting of QHOs having zero-point energy may be classified as "dark", since light propagates in it as a process. The existence of luminiferous medium does not contradict the second postulate of special relativity (the principle of constancy of the velocity of light in inertial systems) if to take into account the interaction of photons with QHOs and with virtual photons (the virtual particles pairs) created by quantum entities that constitute the inertial systems.

Due to possibility of emergence of spin supercurrent between QHOs the background electro-magnetic emission may exist in the physical vacuum consisting of QHOs. The cosmic microwave background (CMB) may be such an emission.

The model of physical vacuum consisting of QHOs considered in this work 
may account for the invisibility of dark matter due to "freezing" of spins of the QHOs at the location of this matter. The "freezing" might take place for example at a large angular speed of rotation of this matter and at low temperature (the latter is characteristic of dark matter).

\section{Acknowledgements}

I am grateful to Mr Mikhail Boldyrev for his assistance in translation of manuscript into English.

\section{Conflicts of Interest}

The author declares no conflicts of interest regarding the publication of this paper.

\section{References}

[1] Peebles, P.J.E. and Ratra, B. (2003) Reviews of Modern Physics, 75, 559-606. https://doi.org/10.1103/RevModPhys.75.559

[2] Chernin, A.D. (2001) Physics Uspekhi, 44, 1099-1118. https://doi.org/10.1070/PU2001v044n11ABEH000962

[3] Matthew, F. (2013) Arstechnica, 22 March 2013.

[4] Einstein, A. and Stern, O. (1913) Annalen der Physik, 345, 551-560. https://doi.org/10.1002/andp.19133450309

[5] Planck, M. (1912) Annalen der Physik, 342, 642-656. https://doi.org/10.1002/andp.19123420403

[6] Puthoff, H.E. (1989) Physical Review A, 40, 4857-4862. https://doi.org/10.1103/PhysRevA.40.4857

[7] Sedov, L.I. (1971-1972) A Course in Continuum Mechanics, Vol. 1-4. Wolters-Noordhof, Groningen.

[8] Boldyreva, L.B. (2018) International Journal of Physics, 6, 128-138.

[9] Purcell, E.M. (1965) Electricity and Magnetism. Berkeley Physics Course, Vol. 2. McGraw-Hill Book Company, New York.

[10] Tsuji, K., Nakamura, S., Sato, T., Kubodera, K. and Myhrer, E. (2004) Physics Letters B, 602, 60-66. https://doi.org/10.1016/j.physletb.2004.09.064

[11] Eidelman, S., et al. (2005) Physics Letters B, 592, 1-5.

[12] Milonni, P.W. (1994) The Quantum Vacuum. Academic Press, Harcourt Brace \& Company Publishers, Cambridge.

[13] Myakishev, G.Y. (1988) Virtual Particles. In: Physics of Microworld. Little Encyclopedia, Soviet Encyclopedia Publishing House, Moscow, 132-133. (In Russian)

[14] Boldyreva, L.B. (2017) International Journal of Physics, 5, 141-146. http://pubs.sciepub.com/ijp/5/4/6/

[15] Boldyreva, L.B. (2014) International Journal of Quantum Information, 12, Article ID: 1450007.

[16] Wichmann, E.H. (1971) Quantum Physics. Berkeley Physics Course, Vol. 4, McGraw-Hill Book Company.

[17] Kaufmann, W. (1902) Physikalische Zeitschrift, 4, 54-56. 
[18] Weber, M.H. and Lynn, K.G. (2000) Radiation Physics and Chemistry, 58, 749-753. https://doi.org/10.1016/S0969-806X(00)00252-8

[19] Borovic-Romanov, A.S., Bunkov, Yu.M., Dmitriev, V.V., Mukharskii, Yu.M. and Sergatskov, D.A. (1989) Physical Review Letters, 62, 1631. https://doi.org/10.1103/PhysRevLett.62.1631

[20] Bunkov, Yu.M. (2009) Journal of Physics. Condensed Matter, 21, Article ID: 164201. https://doi.org/10.1088/0953-8984/21/16/164201

[21] Dmitriev, V.V. and Fomin, I.A. (2009) Journal of Physics: Condensed Matter, 21, Article ID: 164202. https://doi.org/10.1088/0953-8984/21/16/164202

[22] Čerenkov, P.A. (1937) Physical Review, 52, 378. https://doi.org/10.1103/PhysRev.52.378

[23] Boldyreva, L.B. and Sotina, N.B. (1992) Physics Essays, 5, 510-513. https://doi.org/10.4006/1.3029014

[24] Einstein, A. and de Haas, W.J. (1915) Deutsche Physikalische Gesellschaft, Verhandlungen, 17, 152-170.

[25] Einstein, A. (1905) Annalen der Physik, 322, 891-921. https://doi.org/10.1002/andp.19053221004

[26] Fox, J.G. (1965) American Journal of Physics, 33, 1-17. https://doi.org/10.1119/1.1971219

[27] Ritz, W. (1908) Annales de Chimie et de Physique, 13, 145.

[28] Jackson, J.D. (1999) Classical Electrodynamics. 3rd Edition, John Wiley, New York.

[29] Alvager, T. and Barley, J.M. (1964) Physics Letters, 12, 260. https://doi.org/10.1016/0031-9163(64)91095-9

[30] Barnett, S.J. (1936) Reviews of Modern Physics, 135, 129-166.

[31] Sandberg, S.G. (1985) Searl-Effect Generator: Design \& Manufacturing Procedure. School of Engineering \& Applied Sciences, University of Sussex, Falmer.

[32] NASA Science (2015) Dark Energy, Dark Matter. NASA Science: Astrophysics, 5. 